This item was submitted to Loughborough's Research Repository by the author.

Items in Figshare are protected by copyright, with all rights reserved, unless otherwise indicated.

\title{
Airtime usage control in virtualized multi-cell 802.11 networks
}

PLEASE CITE THE PUBLISHED VERSION

http://dx.doi.org/10.1109/GLOCOMW.2015.7414201

PUBLISHER

(c) IEEE

VERSION

AM (Accepted Manuscript)

LICENCE

CC BY-NC-ND 4.0

REPOSITORY RECORD

Derakhshani, Mahsa, Xiaowei Wang, Tho Le-Ngoc, and Alberto Leon-Garcia. 2019. "Airtime Usage Control in Virtualized Multi-cell 802.11 Networks". figshare. https://hdl.handle.net/2134/21337. 


\title{
Airtime Usage Control in Virtualized Multi-Cell 802.11 Networks
}

\author{
Mahsa Derakhshani ${ }^{\dagger}$, Xiaowei Wang*, Tho Le-Ngoc*, Alberto Leon-Garcia ${ }^{\dagger}$ \\ $\dagger$ Department of Electrical \& Computer Engineering, University of Toronto, Toronto, ON, Canada \\ *Department of Electrical \& Computer Engineering, McGill University, Montreal, QC, Canada \\ Email: mahsa.derakhshani@utoronto.ca; xiaowei.wang@mail.mcgill.ca; tho.le-ngoc@mcgill.ca; alberto.leongarcia@utoronto.ca
}

\begin{abstract}
This paper investigates the station (STA)-access point (AP) association and airtime control for virtualized 802.11 networks to provide service customization and fairness across multiple Internet service-providers (ISPs) sharing the common physical infrastructure and network capacity. More specifically, an optimization problem is formulated on the STAs' transmission probabilities to maximize the overall network throughput, while providing airtime usage guarantees for the ISPs. Subsequently, an algorithm to reach the optimal solution is developed by applying monomial approximation and geometric programming iteratively. Illustrative results confirm the superior and robust performance of the developed association and airtime control scheme in terms of both throughput and fairness.
\end{abstract}

\section{INTRODUCTION}

Wireless virtualization has recently emerged as an architectural choice for the wireless networks, in which different service providers can share physical infrastructure and wireless resources. Allowing resources to be shared, vitualization can facilitate a flexible and lower-complexity solution to support customized services with finer control over quality-of-service (QoS) features. To enable service customization, the key issue would be to provide isolation among virtual networks running by different service providers. Such isolation can be achieved through contention-free resource allocation techniques based on TDMA and/or FDMA, by isolating resources across service providers. But, in systems using contention-based access protocols, providing isolation is more challenging.

For instance, in virtualized 802.11 WLANs, transmissions of different virtual WLANs (V-WLANs) are closely coupled, although administrative virtualization (i.e., one physical AP advertises multiple service set identifiers (SSIDs)) can already differentiate groups of flows. With a carrier sense multiple access (CSMA)-based MAC, unavoidable collisions act to couple the transmissions of different V-WLANs. Moreover, since the network capacity is shared yet constrained, the increase of traffic in one V-WLAN may reduce the available network capacity to another [1]. Thus, an efficient resource allocation among V-WLANs is essential to manage the MAClayer couplings.

In a 802.11 WLAN with densely deployed APs, before a STA can access the network, it needs to make a decision about which AP to associate with. In virtualized 802.11 networks, such STA-AP association control could create an opportunity to provide fairness guarantees among different ISPs. In this work, in order to improve the network throughput and enable airtime control among V-WLANs, we aim to explore STA-AP association control.

In most current vendor implementations, 802.11 STAs choose the AP with the highest received signal-to-noise ratio (SNR) to connect with. Since the STA density is often uneven in the network [2], [3], the Max-SNR approach can lead to an unbalanced distribution of STAs among APs, causing unfairness. In order to balance the load of APs, several STA-AP association algorithms have been presented in the literature, mostly by maximizing the minimum throughput of all STAs [4]-[6]. Nevertheless, in a basic service set (BSS) including an AP and its associated multi-rate STAs, it is shown that the throughput is limited by the STA with the lowest data rate. This phenomenon is also refereed to as the performance anomaly problem [7]. Thus, comparing with the Max-SNR approach, these load-balancing approaches improve the max-min fairness among STAs at the cost of decreasing the aggregate throughput.

To address the performance anomaly and balance the tradeoff between aggregate throughput and fairness, proportional fair throughput allocation has widely been considered in multirate 802.11 WLANs [8]-[11]. In [8], proportional fairness is studied in a single BSS. It is shown that propotional fairness leads to an airtime-fairness, where equal airtime usage is provided to all STAs. Moreover, in a multi-AP WLAN, [10], [11] study STA-AP association problem with an objective to maximize the proportional fairness. More precisely, association control is implemented in a form of airtime allocation, where the transmission time of STAs at different APs are jointly optimized [10], [11].

In a virtualized WLAN serving multiple ISPs, STA-AP association and airtime control become more challenging to provide service customization and fairness guarantees for ISPs, while there are unavoidable couplings among the STA transmissions of different ISPs in the network. There are a few works addressing only airtime control in the literature. Considering a virtualized single-AP WLAN, heuristic algorithms [12] and control theory [13] are employed to tune the airtime usage by controlling the minimum contention window $(\mathrm{CW})$ of each STA. But, as the discussion is limited to controlling minimum CW, the optimality of the result might be sacrificed. In [1], a distributed algorithm to allocate airtime slices among 


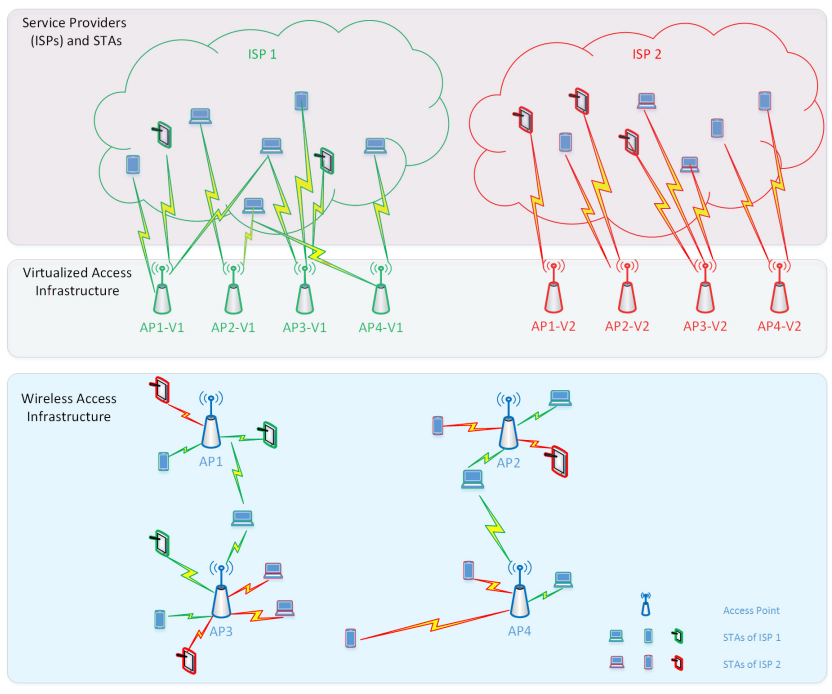

Fig. 1: Layered system model

ISPs and flow rates within each slice in a max-min fair manner is developed for a multi-AP WLAN. Since max-min fairness is used as an objective for rate allocation among the flows in each ISP, the optimality of the achieved throughput may not be guaranteed in a multi-rate WLAN. In addition, the association control is not discussed in [1].

In this work, STA-AP association and airtime control are jointly explored to provide fairness and throughput guarantees for different V-WLANs. Taking into account STA transmission rates and ISP airtime reservations, an optimization problem is formulated to adjust the transmission probability of each STA at each AP. The objective is to maximize the overall network throughput, while keeping a total airtime guarantee for each ISP. To solve the formulated problem which is non-convex and thus computationally intractable, an iterative algorithm is developed by successive geometric programming. This algorithm can achieve an optimal solution with an affordable complexity.

The rest of this paper is organized as follows. Section II presents an overview of the system configuration and modeling. In Section III, we formulate and solve the transmission probability optimization problem, which maximizes the system throughput and guarantees the fairness among the ISPs. Illustrative results are provided in Section IV to evaluate the performance of the developed algorithms. Section V provides some concluding remarks.

\section{System Configuration And Modeling}

We consider an IEEE 802.11-based WLAN that consists of a large number of APs. APs operate on non-overlapping frequency channels. Let $\mathcal{A}$ be the set of APs and $N_{a}=|\mathcal{A}|$ be the total number of APs. Each AP has a limited coverage area and all STAs are randomly distributed in the field. The network carries traffic belonging to a number of different ISPs (also referred to as V-WLANs). Let $\mathcal{K}$ be the set of ISPs using the network. Furthermore, let $\mathcal{S}_{k}$ be the set of STAs of ISP $k \in \mathcal{K}$ and $N_{k}=\left|\mathcal{S}_{k}\right|$ be the number of STAs belong to ISP $k$.
Furthermore, let $\mathcal{S}$ be the set of all STAs and $N_{s}=\sum_{k \in \mathcal{K}} N_{k}$ be the total number of STAs in the network. The network is administratively virtualized, i.e., each AP will broadcast multiple different SSIDs, one for each ISP. Figure 1 illustrates an example of the network architecture with four physical APs and two ISPs.

\section{A. Association Control via Transmission Probabilities}

In a WLAN with APs densely deployed, STAs need to determine which APs to connect with. In a traditional 802.11 WLAN, an STA will choose an AP to associate with based on some criteria (i.e., Max SNR) and then transmit to the AP using CSMA/CA. Due to the random nature of CSMA/CA based protocols, the STAs' access of the channel can be modeled as a random event. Furthermore, the transmission probability of each STA can be calculated based on the network configuration and the MAC parameters used by the STAs [14]. In other words, the transmission probability of the STAs can be controlled by manipulating the MAC layer parameters.

In this work, we aim to generalize the association control problem by adjusting the transmission probability of each STA at any $\mathrm{AP}$, rather than selecting one AP to associate with. Thus, we define $\tau_{i}^{a}\left(0 \leq \tau_{i}^{a} \leq 1\right)$ as the probability that STA $i$ attempts to transmit at AP $a$. Consequently, the probability that a time-slot is idle in the BSS including AP $a$ is

$$
P_{\text {idle }}^{a}=\prod_{i \in \mathcal{S}}\left(1-\tau_{i}^{a}\right) .
$$

In a given BSS, transmitted packets will be received successfully, if exactly one STA transmits on the channel. Thus, the probability of a successful transmission initiated by STA $i$ becomes

$$
P_{\mathrm{succ}, i}^{a}=\tau_{i}^{a} \prod_{i^{\prime} \in \mathcal{S}, i^{\prime} \neq i}\left(1-\tau_{i^{\prime}}^{a}\right) .
$$

According to the 802.11e Enhanced Distributed Access Protocol (EDCA), an STA can transmit multiple back-to-back packets for a fixed period of time. Let $T_{\mathrm{TXOP}}$ be the duration of a data frame. Then, the duration of a successful transmission becomes

$$
T_{s}=T_{\mathrm{TXOP}}+\mathrm{SIFS}+\gamma+\mathrm{ACK}+\gamma+\mathrm{AIFS}
$$

where $\gamma$ denotes the transmission delay; SIFS is the short interframe spacing; ACK is the duration of an acknowledgment; AIFS is the arbitrary inter-frame spacing. Similarly, the duration of a collision can be calculated as

$$
T_{c}=T_{\mathrm{TXOP}}+\gamma+\text { AIFS. }
$$

Since ACK and SIFS are relatively small compared with $T_{\text {TXOP }}$, we approximate $T_{s}$ and $T_{c}$ to be of the same size and denote them by $T$. Consequently, the expected length of a general time-slot becomes

$$
\mathbb{E}\left\{T_{g}\right\}=\delta P_{\text {idle }}^{a}+\left(1-P_{\text {idle }}^{a}\right) T
$$


where $\delta$ is the duration of an idle time-slot. Furthermore, the expected information (in bits) transmitted by STA $i$ to AP $a$ in a general time-slot can be derived as

$$
\mathbb{E}\left\{I_{g}\right\}=P_{\text {succ }, i}^{a} r_{i}^{a} T_{\mathrm{TXOP}}
$$

where $r_{i}^{a}$ represents the transmission data rate of the link between STA $i$ and AP $a$. As defined in [14], based on the (5) and (6), the throughput of STA $i$ at AP $a$ becomes

$$
T_{i}^{a}=\frac{\mathbb{E}\left\{I_{g}\right\}}{\mathbb{E}\left\{T_{g}\right\}}=\frac{P_{\text {succ }, i}^{a} r_{i}^{a} T_{\mathrm{TXOP}}}{P_{\text {idle }}^{a} \delta+\left(1-P_{\text {idle }}^{a}\right) T} .
$$

Let define a new variable $x_{i}^{a}=\frac{\tau_{i}^{a}}{1-\tau_{i}^{a}}\left(x_{i}^{a} \geq 0\right)$, which represents the expected number of consecutive transmission attempts by STA $i$ at AP $a$ as [1], [8]. Consequently, $P_{\text {idle }}^{a}$ and $P_{\text {succ }, i}^{a}$ will be transformed into

$$
\begin{gathered}
P_{\text {idle }}^{a}=\frac{1}{\prod_{i \in \mathcal{S}}\left(1+x_{i}^{a}\right)}, \\
P_{\text {succ }, i}^{a}=\frac{x_{i}^{a}}{\prod_{i^{\prime} \in \mathcal{S}}\left(1+x_{i^{\prime}}^{a}\right)}=x_{i}^{a} P_{\text {idle }}^{a} .
\end{gathered}
$$

Subsequently, from (8) and (9), $T_{i}^{a}$ can be represented in terms of $x_{i}^{a}$ as

$$
T_{i}^{a}=\frac{x_{i}^{a} P_{\mathrm{idle}}^{a} r_{i}^{a} T_{\mathrm{TXOP}}}{T-(T-\delta) P_{\mathrm{idle}}^{a}}=\frac{x_{i}^{a} r_{i}^{a} t}{\prod_{i^{\prime} \in \mathcal{S}}\left(1+x_{i^{\prime}}^{a}\right)-t^{\prime}}
$$

where $t=\frac{T_{\mathrm{TXOP}}}{T}$ and $t^{\prime}=\frac{T-\delta}{T}$.

In addition to the throughput of each STA, the fraction of time that each STA spends for transmission could be considered as another performance metric, specifically in order to measure and preserve fairness among different STAs or ISPs. The total access airtime for STA $i$-including both successful transmissions and collisions-becomes

$$
T_{\mathrm{air}, i}^{a}=\frac{P_{\mathrm{coll}, i}^{a} T+P_{\mathrm{succ}, i}^{a} T}{P_{\text {idle }}^{a} \delta+\left(1-P_{\text {idle }}^{a}\right) T}
$$

where $P_{\text {coll }, i}^{a}=\tau_{i}^{a}\left[1-\prod_{i^{\prime} \in \mathcal{S}, i^{\prime} \neq i}\left(1-\tau_{i^{\prime}}^{a}\right)\right]$ is the probability that STA $i$ suffers from a collision in a general time-slot as defined in [8]. Consequently,

$$
T_{\mathrm{air}, i}^{a}=\frac{\tau_{i}^{a}}{1-P_{\mathrm{idle}}^{a} t^{\prime}}=\frac{x_{i}^{a} \prod_{i^{\prime} \in \mathcal{S}, i^{\prime} \neq i}\left(1+x_{i^{\prime}}^{a}\right)}{\prod_{i^{\prime} \in \mathcal{S}}\left(1+x_{i^{\prime}}^{a}\right)-t^{\prime}} .
$$

In this work, we aim to maximize the overall network throughput, while guaranteeing a minimum requirement on the aggregate airtime of each ISP. To this end, the transmission probability of STAs $\left(\tau_{i}^{a}\right)$ need to be adaptively optimized by maximizing the aggregate throughput of all STAs at all APs (i.e., $\sum_{i \in \mathcal{S}, a \in \mathcal{A}} T_{i}^{a}$ ). Furthermore, for each ISP (e.g., ISP $k$ ), a constraint needs to be set in order to keep the total airtime of all STAs belonging to ISP $k$ larger than a minimum requirement. More specifically, $\sum_{i \in \mathcal{S}_{k}, a \in \mathcal{A}} T_{\mathrm{air}, i}^{a} \geq \eta_{k}$ where $\eta_{k}$ denotes the target share of the airtime for ISP $k$.

\section{OPTIMIZATION PROBLEM}

In this section, we present the STA-AP association and airtime control optimization problem. Based on the system model introduced in Section II, we formulate the optimization problem and solve it by applying monomial approximation and geometric programming.

\section{A. Optimization Problem}

The objective is to maximize the overall network throughput, while distributing access airtime among different ISPs according to their reservations. More specifically, the optimization can be formulated as

$$
\begin{gathered}
\max _{\boldsymbol{X}} \sum_{i \in \mathcal{S}, a \in \mathcal{A}} \frac{x_{i}^{a} r_{i}^{a} t}{\prod_{i^{\prime} \in \mathcal{S}}\left(1+x_{i^{\prime}}^{a}\right)-t^{\prime}}, \quad \text { subject to, } \\
\sum_{i \in \mathcal{S}_{k}, a \in \mathcal{A}} \frac{x_{i}^{a} \prod_{i^{\prime} \in \mathcal{S}, i^{\prime} \neq i}\left(1+x_{i^{\prime}}^{a}\right)}{\prod_{i^{\prime} \in \mathcal{S}}\left(1+x_{i^{\prime}}^{a}\right)-t^{\prime}} \geq \eta_{k}, \quad \forall k \in \mathcal{K}
\end{gathered}
$$

where $X=\left[x_{i}^{a}\right]\left(x_{i}^{a} \geq 0\right)$. The objective function in (13a) represents the overall network throughput (i.e., $\sum_{i \in \mathcal{S}, a \in \mathcal{A}} T_{i}^{a}$ ) based on (10). Constraints in (13b) guarantee the minimum airtime reservations for all ISPs (i.e., $\sum_{i \in \mathcal{S}_{k}, a \in \mathcal{A}} T_{\text {air }, i}^{a} \geq \eta_{k}$ ) based on (12). This set of constraints enable controlling ISPs' share of access airtime regardless of their number of STAs.

The formulated problem is non-convex and thus intractable to solve. However, it potentially looks like an extension of Geometric Programming (GP) (defined in Section VI-A). Thus, by applying successive transformation strategies, we will try to convert the original problem into a series of standard GP problems that can be solved to reach an optimal solution. First, we introduce two auxiliary variables, $y^{a}=$ $\prod_{i^{\prime} \in \mathcal{S}}\left(1+x_{i^{\prime}}^{a}\right)-t^{\prime}, \forall a \in \mathcal{A}$ and $t_{i}^{a}=1+x_{i}^{a}, \forall i \in \mathcal{S}, \forall a \in \mathcal{A}$. Then, the optimization problem in (13) can be transformed into

$$
\begin{aligned}
& \min _{\boldsymbol{X}, \boldsymbol{T}, \boldsymbol{Y}} \sum_{i \in \mathcal{S}, a \in \mathcal{A}}-\frac{x_{i}^{a} r_{i}^{a} t}{y^{a}}, \quad \text { subject to, } \\
& \frac{\prod_{i \in \mathcal{S}}\left(1+x_{i}^{a}\right)}{t^{\prime}+y^{a}}=1, \forall a \in \mathcal{A} \\
& \frac{\eta_{k}+1}{1+\sum_{i \in \mathcal{S}_{k}, a \in \mathcal{A}} \frac{x_{i}^{a} \prod_{i^{\prime} \neq i} t_{i}^{a}}{y^{a}}} \leq 1, \forall k \in \mathcal{K} \\
& \frac{t_{i}^{a}}{1+x_{i}^{a}}=1, \forall i \in \mathcal{S}, a \in \mathcal{A}
\end{aligned}
$$

where $\boldsymbol{T}=\left[t_{i}^{a}\right]\left(t_{i}^{a} \geq 1\right)$ and $\boldsymbol{Y}=\left[y^{a}\right]\left(y^{a}>0\right)$ are the matrices of the corresponding variables. Nevertheless, the transformed problem is not still in a GP form. One reason is that the objective function (14a) is not a posynomial because of negative multiplicative coefficients. To deal with such problem, first, we equivalently substitute the objective function by $\sum_{i \in \mathcal{S}, a \in \mathcal{A}}-x_{i}^{a} r_{i}^{a} t\left(y^{a}\right)^{-1}+M$ where $M$ is a sufficiently large positive constant. Adding $M$ makes sure that the objective function is always positive. Then, we introduce an additional auxiliary variable $x_{0} \geq 0$. By minimizing $x_{0}$ 


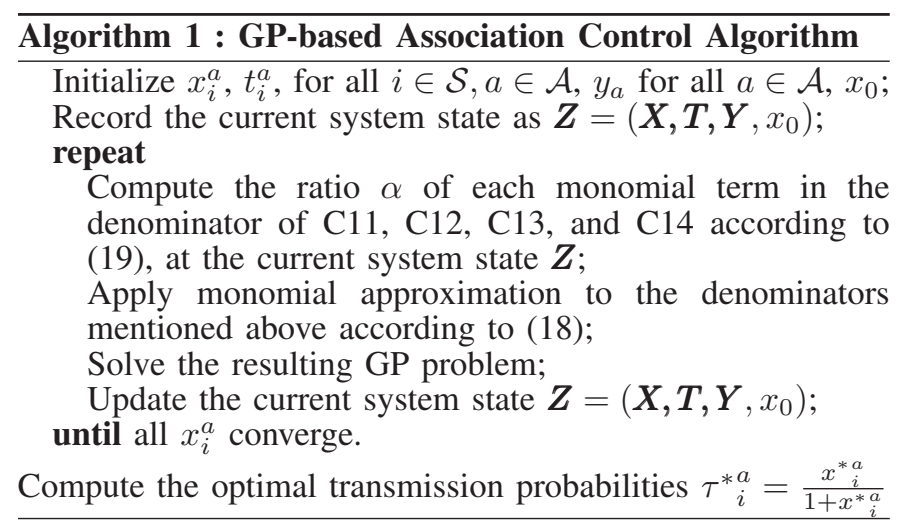

and guaranteeing constraint C11 in (15), we can effectively minimize the objective function in (14a). Consequently,

$$
\begin{aligned}
& \min _{\boldsymbol{X}, \boldsymbol{T}, \boldsymbol{Y}, x_{0}} x_{0}, \quad \text { subject to, } \\
& \mathrm{C} 11: \frac{M}{x_{0}+\sum_{i \in \mathcal{S}, a \in \mathcal{A}}\left(\frac{x_{i}^{a} r_{i}^{a} t}{y^{a}}\right)} \leq 1 \\
& \mathrm{C} 12: \frac{\prod_{i \in \mathcal{S}}\left(1+x_{i}^{a}\right)}{t^{\prime}+y^{a}}=1, \forall a \in \mathcal{A} \\
& \mathrm{C} 13: \frac{\eta_{k}+1}{1+\sum_{i \in \mathcal{S}_{k}, a \in \mathcal{A}} \frac{x_{i}^{a} \prod_{i^{\prime} \in \mathcal{S}, i^{\prime} \neq i} t_{i^{\prime}}^{a}}{y^{a}}} \leq 1, \forall k \in \mathcal{K} \\
& \mathrm{C} 14: \frac{t_{i}^{a}}{1+x_{i}^{a}}=1, \forall i \in \mathcal{S}, a \in \mathcal{A}
\end{aligned}
$$

The optimization problem in (15) belongs to the class of complementary GP problems that allow upper bound constraints on the ratio between two posynomials and equality constraints on the ratio between a monomial and a posynomial [15], [16]. By approximating the posynomials in the denominator of such constraints, a complementary GP can be turned into a standard form of GP. Consequently, the optimal solution can be achieved by iteratively applying monomial approximations and solving a series of GPs. The arithmetic-geometric mean inequality can be used to approximate a posynomial with a monomial. The details of such monomial approximation are provided in Section VI-A.

Accordingly, we propose an iterative algorithm to reach to an optimal solution of the transmission probability optimization problem. In each iteration, monomial approximations are applied to the denominator of $\mathrm{C} 11, \mathrm{C} 12, \mathrm{C} 13$, and $\mathrm{C} 14$. Then, the resulting GP can be solved for instance by using a standard interior-point algorithm. Algorithm 1 presents different steps need to be performed until convergence.

\section{NUMERICAL RESULTS}

In this section, we present numerical results to evaluate the performance of the proposed STA-AP association and airtime control algorithm. More specifically, the performance of our GP-based association scheme is compared with the Max-SNR scheme in terms of throughput and fairness. We used cvx [17] to solve the GP problems in the association algorithm.

\begin{tabular}{|c|c|c|c|}
\hline Modulation & FEC Rate & Data Rate $(\mathrm{Mbps})$ & SNR $(\mathrm{dB})$ \\
\hline BPSK & $1 / 2$ & 6 & {$[5,8)$} \\
\hline BPSK & $3 / 4$ & 9 & {$[8,10)$} \\
\hline QPSK & $1 / 2$ & 12 & {$[10,13)$} \\
\hline QPSK & $3 / 4$ & 18 & {$[13,16)$} \\
\hline 16QAM & $1 / 2$ & 24 & {$[16,19)$} \\
\hline 16QAM & $3 / 4$ & 36 & {$[19,22)$} \\
\hline 64QAM & $2 / 3$ & 48 & {$[22,25)$} \\
\hline 64QAM & $3 / 4$ & 54 & {$[25, \infty)$} \\
\hline
\end{tabular}

TABLE I: IEEE 802.11a adaptive modulation and coding scheme and the SNR ranges used in the numerical results

We consider a network in which 4 APs are deployed in a $10 \times 10 \mathrm{~m}^{2}$ area. More specifically, the APs are placed at the centers of four different $5 \times 5 \mathrm{~m}^{2}$ grids (as shown in Figure 2) to provide seamless coverage. To eliminate interference between the transmission of different APs, four non-overlapping $20 \mathrm{MHz}$ channels are assigned to four APs. The STAs are distributed in the entire area according to the two-dimensional Poisson point process (PPP).

The wireless channel model includes path loss and smallscale fading. Generally, the channel gain can be expressed as $h=A h^{\prime} d^{-\alpha / 2}$, where $d$ is the distance between an STA and an AP, $\alpha \geq 2$ is the path loss exponent, $A$ is a constant dependent on the frequency and transmitter/receiver antenna gain, and $h^{\prime}$ represents the small-scale fading component. In the numerical results, we set $\alpha=3$ and $A=1$. Furthermore, $h^{\prime}$ is randomly generated according to the Rayleigh distribution assuming $\mathbb{E}\left\{\left|h^{\prime}\right|^{2}\right\}=1$. The received SNR at STA $i$ is equal to $\frac{P g_{i}^{a}}{\sigma^{2}}$ where $P$ is the transmission power, $g_{i}^{a}=\left|h_{i}^{a}\right|^{2}$ is the channel power gain from STA $i$ to AP $a$, and $\sigma^{2}$ is the noise power. In the numerical results, $P / \sigma^{2}$ is assumed to be $10 \mathrm{~dB}$.

To determine the transmission rate of each STA-AP pair, the 802.11a adaptive modulation and coding is used based on the received SNR as indicated in Table I.

The MAC layer parameters used in our simulations are set as follows: idle time slot $\delta=9 \mu \mathrm{s}$, propagation delay $\gamma=$ $1 \mu \mathrm{s}$, fixed transmission duration $T_{\mathrm{TXOP}}=1 \mathrm{~ms}$, short interframe space SIFS $=10 \mu \mathrm{s}$, acknowledgment $\mathrm{ACK}=40 \mu \mathrm{s}$. Moreover, the target airtime share for each ISP $k$ (i.e., $\eta_{k}$ ) is set equal to the number of APs divided by the number of ISPs. In other words, we assume that the ISPs have the same minimum airtime reservation and share the total airtime in a fair manner.

\section{A. Sample Association Result}

Figures 2 and 3 illustrate how the proposed GP-based STAAP association scheme works in comparison with Max-SNR scheme. In these examples, we consider two ISPs, while ISP 1 (green with circles) is serving 2 STAs and ISP 2 (red with stars) is serving 4 STAs. Comparing Figures 2 and 3, it is clear that GP-based scheme assigns larger transmission probabilities to STAs of the ISP with smaller number of STAs (i.e, green with circles). Thus, it is able to improve the fairness between two ISPs compared with the Max-SNR. GP-based scheme 


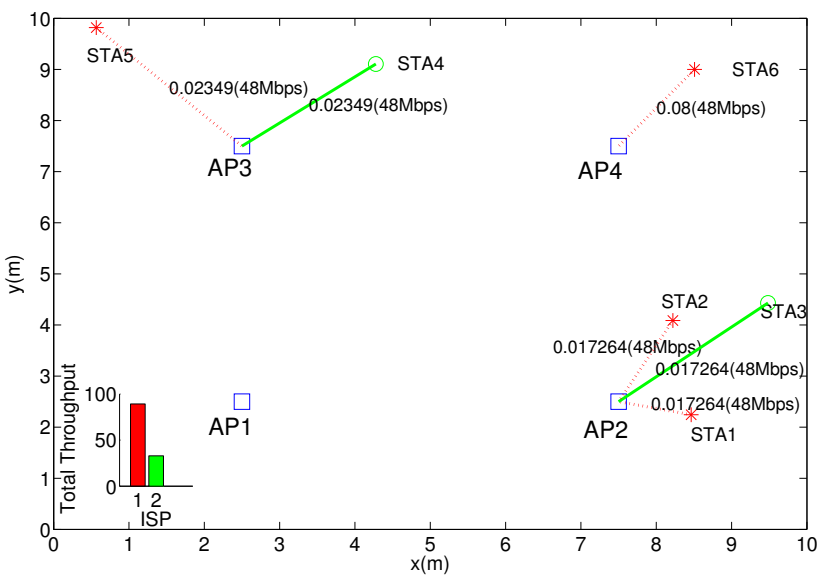

Fig. 2: Association result: Max-SNR association control

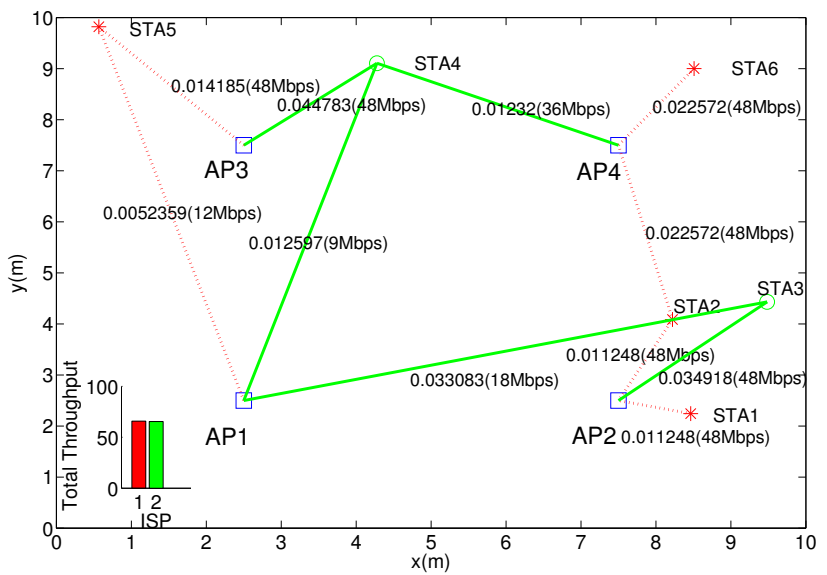

Fig. 3: Association result: The proposed GP-based association control
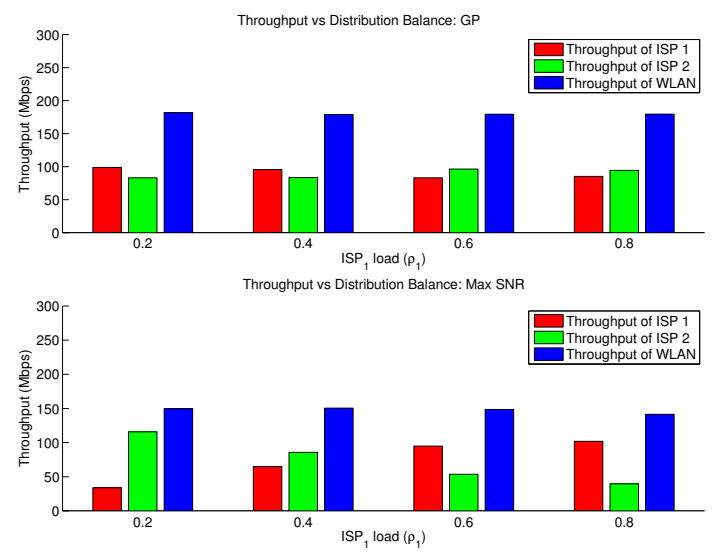

Fig. 4: Throughput vs. ISP 1 load

can also balance the load of the APs, which can potentially improve the overall network throughput.

\section{B. Effects of STA Density and ISP Load}

Let define $\rho_{1}$ as the ratio of number of STAs serving by ISP 1 to the total number of STAs in the network (also referred to

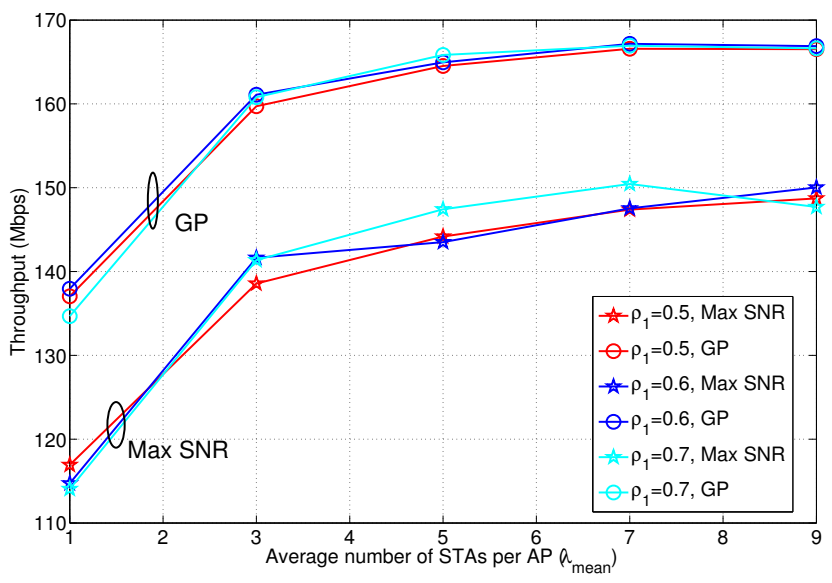

Fig. 5: Total throughput vs. STA density for different $\rho_{1}$

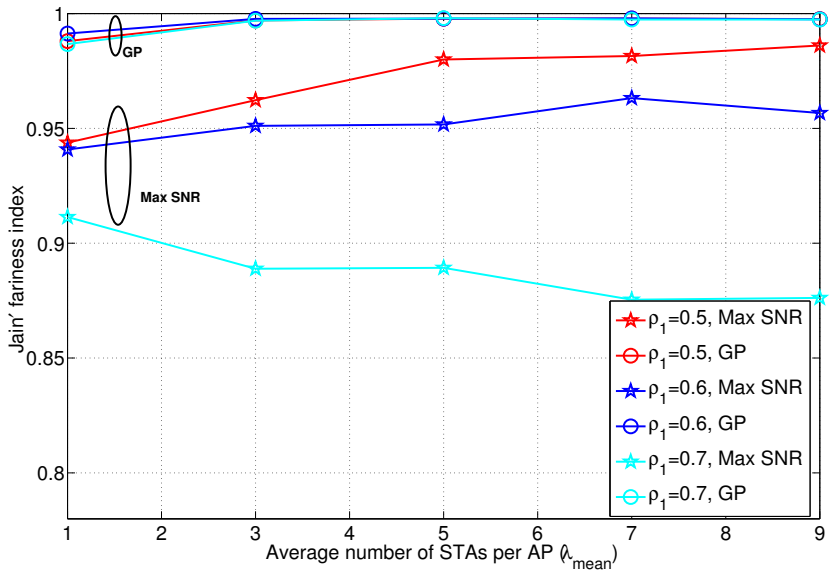

Fig. 6: Fairness vs. STA density for different $\rho_{1}$

as ISP 1 load). Here, the performance of the two association approaches are compared for different STA density and ISP load.

Assuming a homogeneous STA distribution with $\lambda_{\text {mean }}=3$, Figure 4 demonstrates the achieved throughput of two ISPs versus different values of $\rho_{1}$ for both GP-based and MaxSNR association schemes. By Max-SNR association, it is shown that throughput of ISP 1 grows linearly with $\rho_{1}$, while the achieved throughput of ISP 2 is decreasing. But, GPbased association can fairly distribute the airtime between ISPs regardless of their ISP loads and thus keep a perfect balance between the achieved throughput of the two ISPs.

Figure 5 shows the total throughput achieved by the two association algorithms versus the average number of STAs per AP, $\lambda_{\text {mean }}$, for a homogeneous STA distribution. For a fixed $\rho_{1}$, the total throughput by both algorithms increases with the STA density (i.e., $\lambda_{\text {mean }}$ ). But, their throughput increase rate is reduced with higher $\lambda_{\text {mean }}$. This is because the wireless channel is underutilized at low STA density. Thus, increasing in the STA density will improve the total throughput. But, when the STA density is sufficiently high, increasing the STA density further will result in a higher collision probability, and hence, slow down the total throughput improvement. For any 
fixed $\rho_{1}$, it is shown that GP-based association significantly improves the total throughput as compared with the Max-SNR association.

The fairness is considered in terms of the Jain's fairness index

$$
F=\frac{\left(\sum_{k \in \mathcal{K}} T_{k}\right)^{2}}{|\mathcal{K}| \sum_{k \in \mathcal{K}} T_{k}^{2}}
$$

where $T_{k}=\sum_{i \in S_{k}, a \in A} T_{i}^{a}$ is the achieved throughput for all the STAs of ISP $k$. Figure 6 indicates that the proposed GP-based association scheme can guarantee perfect fairness between the ISPs with a fairness index approaching 1, over a wide range of STA density and ISP 1 load. The achieved fairness level by Max-SNR association is worse than GPbased, especially when the STA load is highly unbalanced between ISPs (i.e., $\rho_{1}$ is not close to 0.5).

\section{CONCLUSION}

This paper considers the STA-AP association and airtime control in virtualized 802.11 networks aiming to provide high throughput and fairness guarantees among ISPs despite the number of STAs per ISP. An optimization problem on the transmission probability of each STA is formulated, which can maximize the network throughput, while guaranteeing the airtime usage of different ISPs. The optimal transmission probabilities are obtained by successive monomial approximation and geometric programming. Extensive numerical results confirm that the proposed association algorithm can improve the throughput and provide fairness guarantees in virtualized 802.11 WLANs.

\section{APPENDIX}

\section{A. Geometric Programming and Monomial Approximation}

An optimization problem is called geometric programming if it follows the following form,

$$
\begin{aligned}
& \min _{\boldsymbol{x}} f_{0}(\boldsymbol{x}), \quad \text { subject to } \\
& f_{i}(\boldsymbol{x}) \leq 1, i=1, \ldots, n_{1} \\
& g_{i}(\boldsymbol{x})=1, i=1, \ldots, n_{2}
\end{aligned}
$$

where $f_{0}, \ldots, f_{n_{1}}$ are posynomials and $g_{1}, \ldots, g_{n_{2}}$ are monomials. In the context of geometric programming, a monomial function $f$ of $\boldsymbol{x}=\left(x_{1}, x_{2}, \ldots, x_{n}\right)$ is defined as,

$$
f(\boldsymbol{x})=c x_{1}^{a_{1}} x_{2}^{a_{2}} \ldots x_{n}^{a_{n}}
$$

where $c>0$ and $a_{i} \in \mathcal{R}$. Furthermore, a posynomial is defined as the summation of multiple monomials, i.e.,

$$
g(\boldsymbol{x})=\sum_{k=1}^{K} f_{k}(\boldsymbol{x})
$$

The basic idea of monomial approximation is as follows: consider a posynomial function $g(\boldsymbol{x})=\sum_{k} f_{k}(\boldsymbol{x})$ with $f_{k}(\boldsymbol{x})$ being the monomial terms. By the arithmetic-geometric mean inequality, we have

$$
g(\boldsymbol{x}) \geq \hat{g}(\boldsymbol{x})=\prod_{k}\left(\frac{f_{k}(\boldsymbol{x})}{\alpha_{k}\left(\boldsymbol{x}_{0}\right)}\right)^{\alpha_{k}\left(\boldsymbol{x}_{0}\right)}
$$

where the parameters $\alpha_{k}\left(\boldsymbol{x}_{0}\right)$ can be obtained by computing

$$
\alpha_{k}\left(\boldsymbol{x}_{0}\right)=\frac{f_{k}\left(\boldsymbol{x}_{0}\right)}{g\left(\boldsymbol{x}_{0}\right)}, \forall k
$$

where $x_{0}>0$ is a fixed point (e.g., the optimal solution from the last round of optimization). It is proved that $\hat{g}(\boldsymbol{x})$ is the best local monomial approximation of $g(x)$ near $x_{0}$ [18].

\section{REFERENCES}

[1] A. Checco and D. Leith, "Fair virtualization of 802.11 networks," IEEE/ACM Trans. Netw., vol. 23, no. 1, pp. 148-160, Feb 2015.

[2] M. Balazinska and P. Castro, "Characterizing mobility and network usage in a corporate wireless local-area network," in Proc. of the 1st Intl. Conf. on Mobile Systems, Applications and Services., May 2003, pp. 303-316.

[3] D. Schwab and R. Bunt, "Characterising the use of a campus wireless network," in Proc. IEEE Intl. Conf. on Computer Commun. (INFOCOM), Mar. 2004, pp. 862-870.

[4] H. Gong, K. Nahm, and J. W. Kim, "Distributed fair access point selection for multi-rate IEEE 802.11 WLANs," in Proc. IEEE Consum. Commun. and Netw. Conf., Jan. 2008, pp. 528-532.

[5] O. Ercetin, "Association games in IEEE 802.11 wireless local area networks," IEEE Trans. Wireless Commun., vol. 7, no. 12, pp. 51365143, Dec. 2008

[6] W. Xu, C. Hua, and A. Huang, "A game theoretical approach for load balancing user association in 802.11 wireless networks," in Proc. IEEE Global Commun. Conf. (GLOBECOM), Dec. 2010, pp. 1-5.

[7] M. Heusse, F. Rousseau, G. Berger-Sabbatel, and A. Duda, "Performance anomaly of $802.11 \mathrm{~b}$," in Proc. IEEE Intl. Conf. on Computer Commun. (INFOCOM), Mar. 2003, pp. 836-843.

[8] A. Checco and D. Leith, "Proportional fairness in 802.11 wireless LANs," IEEE Commun. Lett., vol. 15, no. 8, pp. 807-809, Aug. 2011.

[9] X. Chen and D. Leith, "Proportional fair coding for 802.11 WLANs," IEEE Commun. Lett., vol. 1, no. 5, pp. 468-471, Oct. 2012.

[10] S.-C. Liew and Y. Zhang, "Proportional fairness in multi-channel multirate wireless networks - Part I: The case of deterministic channels with application to AP association problem in large-scale WLAN," IEEE Trans. Wireless Commun., vol. 7, no. 9, pp. 3446-3456, Sept. 2008.

[11] Y. Zhang and S.-C. Liew, "Proportional fairness in multi-channel multirate wireless networks - Part II: The case of time-varying channels with application to OFDM systems," IEEE Trans. Wireless Commun., vol. 7, no. 9, pp. 3457-3467, Sept. 2008.

[12] K. Nakauchi, Y. Shoji, and N. Nishinaga, "Airtime-based resource control in wireless LANs for wireless network virtualization," in Fourth Intl. Conf. on Ubiquitous and Future Networks (ICUFN),, July 2012, pp. 166-169.

[13] A. Banchs, P. Serrano, P. Patras, and M. Natkaniec, "Providing throughput and fairness guarantees in virtualized WLANs through control theory," Mobile Networks and Applications., vol. 17, no. 4, pp. 435446, June 2012.

[14] G. Bianchi, "Performance analysis of the IEEE 802.11 distributed coordination function," IEEE Journal on Selected Areas in Communications, vol. 18, no. 3, pp. 535-547, Mar 2000.

[15] M. Chiang, Geometric programming for communication systems. Now Publishers Hanover, 2005.

[16] G. Xu, "Global optimization of signomial geometric programming problems," European Journal of Operational Research, vol. 233, no. 3, pp. 500-510, 2014.

[17] M. Grant and S. Boyd, "CVX: Matlab software for disciplined convex programming, version 2.1," http://cvxr.com/cvx, Mar. 2014.

[18] S. Boyd and L. Vandenberghe, Convex Optimization. Cambridge University Press, 2004. 